\title{
A good beginning with an uncertain future
}

\author{
Heinrich R. Schelbert, MD, PhD, ${ }^{a}$ and Magnus Dahlbom, $\mathrm{PhD}^{\mathrm{a}}$ \\ a Department of Molecular and Medical Pharmacology, David Geffen School of Medicine at \\ UCLA, University of California at Los Angeles, Los Angeles, CA
}

Received Sep 1, 2017; accepted Sep 1, 2017

doi: 10.1007/s12350-017-1091-9

\section{See related article, pp. 798-809}

The capability to evaluate myocardial perfusion and to measure extent and severity of perfusion defects noninvasively and repeatedly in small animals holds considerable promise for exploring the effectiveness of cardioprotective therapeutic strategies. Several investigations have shown that this is possible indeed with dedicated radionuclide imaging devices and radiotracers of myocardial blood flow and substrate metabolism. ${ }^{1-6}$ Non-invasively observed regional radiotracer tissue concentrations were found to be closely correlated with those measured postmortem by tissue counting. Image-derived sizes of perfusion defects closely matched the infarct sizes determined postmortem by tissue staining techniques and thus were highly accurate. ${ }^{1}$ These investigations employed radiotracers like Tc-99 m sestamibi, ${ }^{2,5} \mathrm{~N}-13$ ammonia, ${ }^{1} \mathrm{~F}-18$ deoxyglucose, and F-18 flurpiridaz. ${ }^{6} \mathrm{~A}$ new investigation published in the current issue of the Journal $^{7}$ adds rubidium-82 as another radiotracer for imaging the myocardium in small animals. Rubidium- 82 has become firmly established as a clinically useful radiotracer of myocardial blood flow. Despite shortcomings related to its physical and biologic properties, the authors argue convincingly in support of rubidium- 82 for myocardial perfusion imaging in small animals because of (a) its now wide availability for clinical myocardial perfusion imaging and quantitative blood flows without the need for a cyclotron; (b) the ease of use and its low cost, that is, the use of rubidium- 82 for animal experimental

Reprint requests: Heinrich R. Schelbert, MD, PhD, Department of Molecular and Medical Pharmacology, David Geffen School of Medicine at UCLA, University of California at Los Angeles, B2085J; Box 95648, 650 Charles E. Young Drive South, Los Angeles, CA 90095-6948; hschelbert@mednet.ucla.edu

J Nucl Cardiol 2019;26:810-3.

$1071-3581 / \$ 34.00$

Copyright (c) 2017 American Society of Nuclear Cardiology. studies after expiration of the strontium-82/rubidium- 82 generator system for clinical use; and (c) the possibility of short-term repeat perfusion measurements due to its ultrashort physical halftime.

The study explores the feasibility of myocardial perfusion imaging and of estimating the size of perfusion defects with rubidium-82 in 29 male SpragueDawley rats; 18 rats undergo a permanent proximal coronary occlusion whereas 11 animals are submitted to a 35-minute transient coronary occlusion followed by reperfusion. PET and MR images are obtained one day prior to and one day after the coronary ligation. Left ventricular volumes together with wall motion and thickening are derived from gated MR images and the infarct size is estimated from the late-enhancing enddiastolic myocardial images. Processing and analysis of the rubidium-82 image data include re-orientation into short, vertical, and horizontal long axis images and operator-assisted semi-automated assembly of polar maps for assessing regional myocardial radiotracer concentrations in a standard 17-segment heart model.

Pre- and post-occlusion rubidium-82 PET myocardial perfusion images shown in the manuscript are of moderate to good diagnostic quality. Experimentally induced perfusion defects are well appreciated on the images and typically involve the anterior wall and the apex. The images support the authors' claim that perfusion imaging with rubidium-82 in small animals is indeed feasible and potentially useful for visualizing regional myocardial perfusion defects. As expected, permanent coronary ligations produced larger infarctions than transient occlusions as measured on the lateenhancing myocardial lesions on MRI $(34.7 \%$ vs. $27 \%$ myocardium). The size of infarcted myocardium correlated inversely with MRI-derived left ventricular ejection fractions. Moreover, infarct sizes were negatively associated with total myocardial uptake of rubidium-82 uptake as determined from the perfusion images. Yet, this decrease in total radiotracer uptake was only modest, averaging only $10.9 \%$ in the permanent and only $3.7 \%$ in the transient occlusion group. On the 
post-occlusion images, regional radiotracer concentrations were lowest in the apical segments, with average values of only $14.3 \%$ and $12.2 \%$ lower than in basal and, presumably, normal myocardial segments. Judging from scatter plots included in the manuscript, individual segments exhibited more severe reductions. Yet these reductions were modest, not exceeding $40 \%$ to $50 \%$ even in myocardial regions with transmural infarcts on late-enhancement MR. Importantly, the study does not present quantitative estimates of infarct sizes derived from rubidium-82 PET images.

The observed only mild or, at best, moderate reductions in radiotracer uptake in infarct regions differ from those observed with Tc-99m sestamibi SPECT and N-13 ammonia, F-18 deoxyglucose or F-18 flurpiridaz PET in earlier studies. Permanent occlusions in these studies produced severe reductions in regional radiotracer uptake, to levels as low as blood pool activity in some. Importantly, image-derived infarct sizes in these earlier investigations correlated closely with those determined post mortem by tissue staining, some with slopes of the regression line at or approaching unity. The findings of the current study are difficult to reconcile with those reported previously using radiotracers of flow other than rubidium-82. Yet, possibilities that might explain the underestimation of the true defect severity and extent include (a) insufficient correction for scatter and promptgamma events; (b) non-gated image acquisition; and (c) the positron range of rubidium- 82 .

(a) Correction routines for scatter, random, and promptgamma events are fully incorporated into image reconstruction algorithms of modern PET systems as used in the current study. Their accuracy has been well established, especially for commonly used positron emitting radionuclides like F-18, C-11, and $\mathrm{N}-13$. Insufficient correction for these events is thus unlikely to account for the underestimation of defect severity but cannot be fully excluded, given the complex physical properties of rubidium- 82 .

(b) Gated acquisition of perfusion images would have reduced the blurring effect of cardiac motion and thus might have led to more precise delineation of perfusion defects and more realistic estimates of reductions in regional radiotracer activity. The authors did explore the possibility of gating but refer to the poor diagnostic quality of gated rubidium-82 perfusion images. Administration of higher radioactivity doses might have improved the image quality. However, in view of the low specific activity of rubidium- 82 in the eluate of the aged generator system, it would have required injection of prohibitively large eluate volumes that are not tolerated by the small animals. (c) The positron range of rubidium-82 appears to have been a major source for underestimating the severity of perfusion defects. Positrons typically do not annihilate with an electron at the site of their initial deposition but travel until losing energy and then annihilate into two $511 \mathrm{keV}$ gamma photons. ${ }^{8}$ The distance of travel varies between different positron emitting radionuclides and depends on the positron's initial kinetic energy and on its interaction with tissue. For example, positrons travel farther in low density (i.e., lung) than in higher density tissues (i.e., bone, myocardium).

Rather than following a Gaussian function, the distribution of "positron flights", as the linear distances between the point of initial deposition and the point of annihilation reflects a narrow peak with wide tails. Monte Carlo-based simulations of positron flights in human tissues yield a positron range of $7.6 \mathrm{~mm}$ (fullwidth tenth-maximum, FWTM) for rubidium-82 in soft tissue like myocardium and substantially shorter ranges as, for example, of $0.91 \mathrm{~mm}$ FWTM for fluorine-18 and of $2.12 \mathrm{~mm}$ FWTM for nitrogen-13. ${ }^{9}$ Besides degrading the spatial resolution, the long positron flight leads to a "cloud of annihilation points around the positron source,' or in the case of myocardial perfusion imaging, around the heart. It seems like such cloud of annihilation events can be appreciated visually as a light-to-dark blue halo surrounding the myocardium on the images.

The long positron range of rubidium-82 has considerable implications for myocardial perfusion imaging for several reasons: besides degrading the image quality, it leads to substantial partial volume related decreases in observed radiotracer tissue concentrations ${ }^{10}$ and to scatter or spillover of activity into flow defects with contamination of true regional tissue radioactivity concentrations. In humans, the long positron range of rubidium-82 does in fact lower the image quality as noted by loss of crispness of anatomical display which becomes especially obvious when compared to images obtained with N-13 ammonia or F-18 flurpiridaz. Yet, the degree of image degradation does not appear to negatively affect the diagnostic accuracy of perfusion images in humans. In small animals however with left ventricles of only $6 \mathrm{~mm}$ in diameter and 1.5-mm-thick myocardium, the range of positrons may prove to be prohibitive for measurements of regional myocardial radiotracer concentrations and for assessing extent and severity of perfusion defects. The enclosed Figure 1 shows simulation studies to demonstrate the degrading effects of different positron ranges on the quality of myocardial images in rats. The long rubidium-82 positron range dramatically lowers the visualized myocardial activity concentration together with substantial blurring of the 

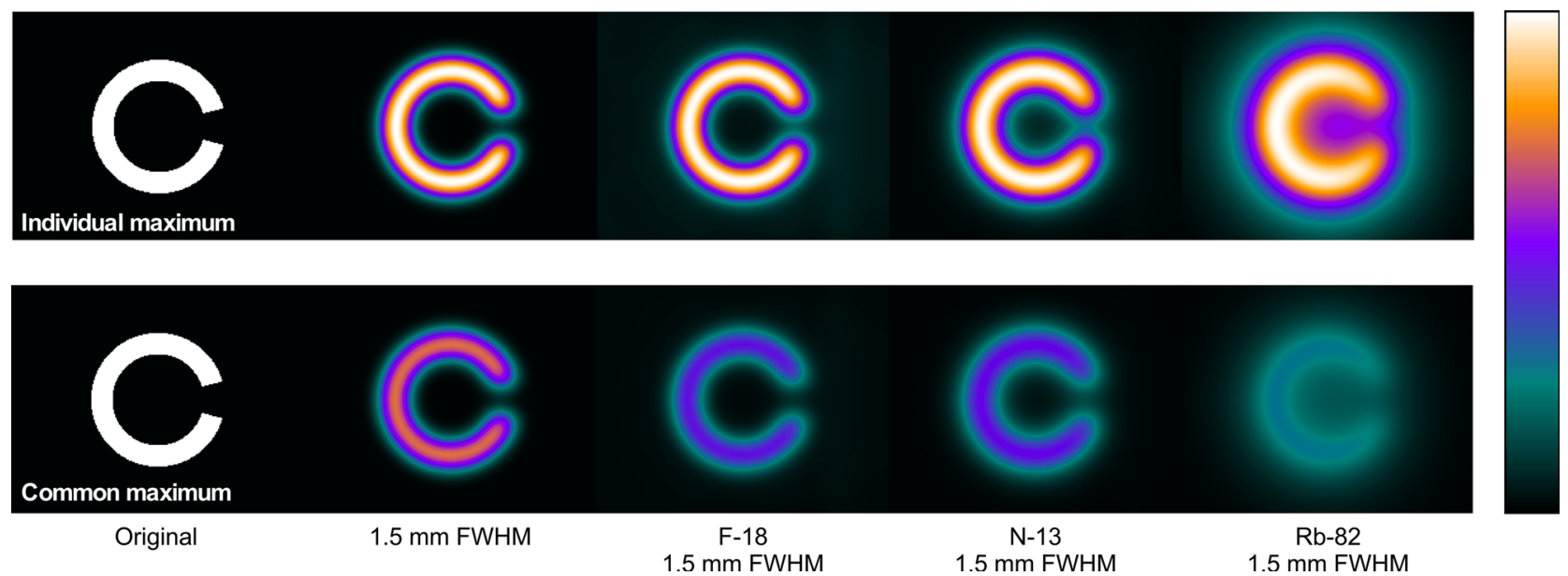

Figure 1. Effects of limited spatial resolution and positron range of a simulated rat heart with a defect. From left to right: true activity distribution $(6 \mathrm{~mm}$ ventricle diameter, $1.5 \mathrm{~mm}$ wall thickness, $2 \mathrm{~mm}$ defect); effect of limited instrument spatial resolution (1.5 mm FWHM); effect of the $\mathrm{F}-18$ positron range in addition to the limited spatial resolution; $\mathrm{N}-13$ positron range in addition to the limited spatial resolution; Rb-82 positron range in addition to the limited spatial resolution. All images were scaled to individual maximum in the top row. The images in the bottom row were scaled to a common maximum (the maximum of the original activity distribution). Data from ${ }^{8}$ and $^{9}$ were used to simulate the effect of the positron range.

perfusion defect. It is thus likely that most the underestimation of the defect severity as reported in the current study can be ascribed to the long positron range that effectively limits quantitative assessments of flow defect sizes and severity in the rat heart.

The current study on rubidium-82 myocardial perfusion imaging is indeed "'a good beginning", as it convincingly demonstrates the feasibility of myocardial perfusion imaging with rubidium-82 in the small hearts of rats with adequate visualization of experimentally induced perfusion defects. Doubt, however, remains whether limitations imposed by the complex and unique physical properties of rubidium- 82 can be adequately resolved so that the myocardial uptake of the radiotracer and the size of experimentally induced flow defects can be measured more accurately. Until then, the value of the described imaging approach for assessing responses to cardioprotective therapeutic strategies remains questionable and its future uncertain.

\section{References}

1. Kudo T, Fukuchi K, Annala AJ, Chatziioannou AF, Allada V, Dahlbom M, et al. Noninvasive measurement of myocardial activity concentrations and perfusion defect sizes in rats with a new small-animal positron emission tomograph. Circulation. 2002;106:118-23.
2. Liu Z, Kastis GA, Stevenson GD, Barrett HH, Furenlid LR, Kupinski MA, et al. Quantitative analysis of acute myocardial infarct in rat hearts with ischemia-reperfusion using a high-resolution stationary SPECT system. Journal of Nuclear Medicine : Official Publication, Society of Nuclear Medicine. 2002;43:933-9.

3. Inubushi M, Jordan MC, Roos KP, Ross RS, Chatziioannou AF, Stout DB, et al. Nitrogen-13 ammonia cardiac positron emission tomography in mice: Effects of clonidine-induced changes in cardiac work on myocardial perfusion. European Journal of Nuclear Medicine and Molecular Imaging. 2004;31:110-6.

4. Higuchi T, Nekolla SG, Huisman MM, Reder S, Poethko T, Yu M, et al. A new 18F-labeled myocardial PET tracer: Myocardial uptake after permanent and transient coronary occlusion in rats. Journal of Nuclear Medicine: Official Publication, Society of Nuclear Medicine. 2008;49:1715-22.

5. Thomas D, Bal H, Arkles J, Horowitz J, Araujo L, Acton PD, et al. Noninvasive assessment of myocardial viability in a small animal model: Comparison of MRI, SPECT, and PET. Magnetic Resonance in Medicine. 2008;59:252-9.

6. Sherif HM, Saraste A, Weidl E, Weber AW, Higuchi T, Reder S, et al. Evaluation of a novel (18)F-labeled positron-emission tomography perfusion tracer for the assessment of myocardial infarct size in rats. Circulation Cardiovascular Imaging. 2009;2:77-84.

6. Ghotbi AA, Clemmensen A, Kyhl K, Follin B, Hasbak P, Engstrom T, et al. Rubidium-82 PET imaging is feasible in a rat myocardial infarction model. Journal of Nuclear Cardiology: Official Publication of the American Society of Nuclear Cardiology. 2017. doi:10.1007/s12350-017-0994-9.

8. Levin CS, Hoffman EJ. Calculation of positron range and its effect on the fundamental limit of positron emission tomography system spatial resolution. Physics in Medicine and Biology. 1999;44:78199. 
9. Sanchez-Crespo A, Andreo P, Larsson SA. Positron flight in human tissues and its influence on PET image spatial resolution. European Journal of Nuclear Medicine and Molecular Imaging. 2004;31:44-51.
10. Hoffman EJ, Huang SC, Phelps ME. Quantitation in positron emission computed tomography: 1. Effect of object size. Journal of Computer Assisted Tomography. 1979;3:299-308. 